\title{
Emergency Bedside Ultrasound Diagnosis of Aortic Dissection in a Young Patient with Biliary Colic
}

\author{
Muhammad Waseem*, Brian Scallen, Viraj Lakdawala, Yocheved Rose and Fernando Jara \\ Department of Emergency Medicine, Lincoln Medical \& Mental Health Center, 234 East $149^{\text {th }}$ Street, Bronx, NY 10451, \\ USA
}

\begin{abstract}
Background: Aortic dissection is a potentially fatal condition that primarily affects the older population. Rapid diagnosis of dissection is crucial for a favorable outcome and the only effective strategy to improve outcome is high clinical suspicion and prompt treatment.

Objective: We present a case that demonstrates a timely diagnosis of a serious and life-threatening condition by an emergency bedside ultrasound (EBU).

Case Report: We report a case of a 36-year-old male with prior history of cholelithiasis and frequent ED visits for biliary colic that presented with abdominal pain. An emergency bedside ultrasound showed gallstones and an aortic dissection in the abdominal aorta. Emergent surgical consultation was obtained based on the ultrasound finding, and subsequently followed with confirmatory imaging and surgical intervention.

Conclusion: This case report highlights the utility of emergency bedside ultrasound, which made a timely diagnosis and saved a life.
\end{abstract}

Keywords: Aortic aneurysm, aortic dissection, dissecting aneurysm, abdominal pain, Emergency bedside Ultrasound.

\section{INTRODUCTION}

Dissection of the aorta is a serious event with poor outcome. This is a life-threatening condition that requires rapid recognition and possibly emergent surgical intervention. Presence of a prior condition with the potential to cause abdominal pain can be misleading when the chief complaint is abdominal pain. With widespread availability and use of emergency bedside ultrasound, certain diagnoses can be quickly established. Emergency bedside ultrasound has now become part of the standard of care for several indications including aortic emergencies.

\section{CASE PRESENTATION}

A 36-year-old male visited the Emergency Department (ED) with a history of epigastric and right upper quadrant abdominal pain for the past twelve hours. The patient was extremely agitated and reported the pain as sharp, constant and radiating to his back. He felt nauseated and vomited twice prior to his presentation in the ED. The patient denied fever, cough, chest pain, shortness of breath, or back pain. His past medical history was significant only for cholelithiasis. The past family history was unknown. He reported his current pain was similar to previous episodes of biliary colic. His only medication was acetaminophen/codeine as needed for pain. He denied alcohol or substance abuse.

On arrival his vital signs were as follows: Heart rate 66 beats/minute, respiratory rate 16 breaths/minute, blood

*Address correspondence to this author at the Department of Emergency Medicine, Lincoln Medical \& Mental Health Center, 234 East $149^{\text {th }}$ Street, Bronx, NY 10451, USA; Tel: (718) 579-6010; Fax: (718)579-4822;

E-mail: waseemm2001@hotmail.com pressure $157 / 70 \mathrm{~mm} \mathrm{Hg}$, temperature 37 degrees Celsius, and oxygen saturation $96 \%$ on room air. On physical examination, he appeared uncomfortable, had moist mucous membranes. Chest examination included pectus excavatum and clear bilateral breath sounds. Heart sounds were normal and no murmurs were appreciated. Abdominal exam revealed epigastric and right upper quadrant abdominal tenderness without rebound tenderness, rigidity or involuntary guarding. There were no surgical scars, distention, palpable abdominal masses or pulsations, and bowel sounds were present in all quadrants. There was no costovertebral angle tenderness, genital examination was normal and hernial orifices were intact.

While awaiting a computed tomography scan, the physicians performed an emergency bedside abdominal ultrasound, with the intention of imaging his gallbladder to rule out cholecystitis. The ultrasound revealed an unexpected finding of aortic dissection in the abdominal aorta (Fig. 1). A CT scan of chest and abdomen (Fig. 2a, $\mathbf{b}$ and $\mathbf{c}$ ) revealed dissection of the entire aorta from the aortic root to just superior to the renal bifurcation and aortic root dilatation.

A continuous labetalol infusion was initiated and the systolic blood pressure was titrated to $120 \mathrm{~mm} \mathrm{Hg}$ and heart rate to 60 beats/min. He subsequently underwent successful surgical repair of the aortic dissection and aortic valve replacement. He was discharged home in stable condition.

\section{DISCUSSION}

Abdominal pain is a common presentation in the emergency department. Aortic dissection is more likely to occur in elderly males and less likely to occur before age 40 [1]. The peak age of occurrence is between 60 and 80 years but 


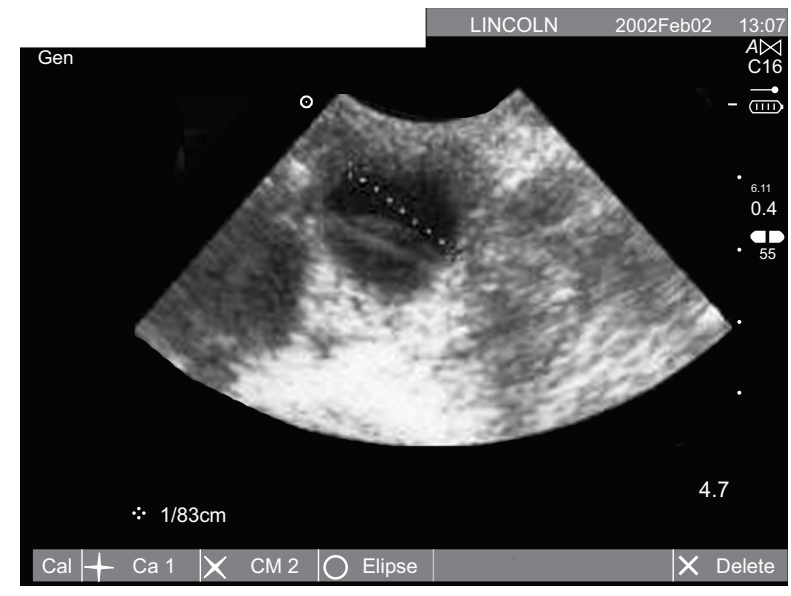

Fig. (1). Bedside ultrasound depicting intimal flap and intramural thrombus.

can occur in relatively young patients especially in the presence of risk factors [2]. Physicians are less likely to consider these diagnoses in a younger patient without major risk factors [3]. Emergency physicians always consider lifethreatening conditions first, however in patients with preexisting conditions, these causes may not be initially considered as a potential cause of abdominal pain due to the confounding previous history.

Aortic dissections are classified in two systems: the Debakey and Stanford classification systems. The Debakey system classifies dissections in one of three classes: type I, II, III. Type I involves the ascending and descending aorta, type II involves the ascending aorta only, and type III involves the descending aorta only [4]. The Stanford classification is divided into two groups - types A and B. Type A is any dissection that involves the ascending aorta, while type $\mathrm{B}$ dissections occur distal to the left subclavian artery [5].

Acute aortic dissection is a potentially catastrophic condition with significant mortality. It is fatal if not recognized early and treated with aggressive medical and/or surgical therapy. Overall in-hospital mortality for all acute aortic dissections is reported to be $27 \%$. When Type A dissections are

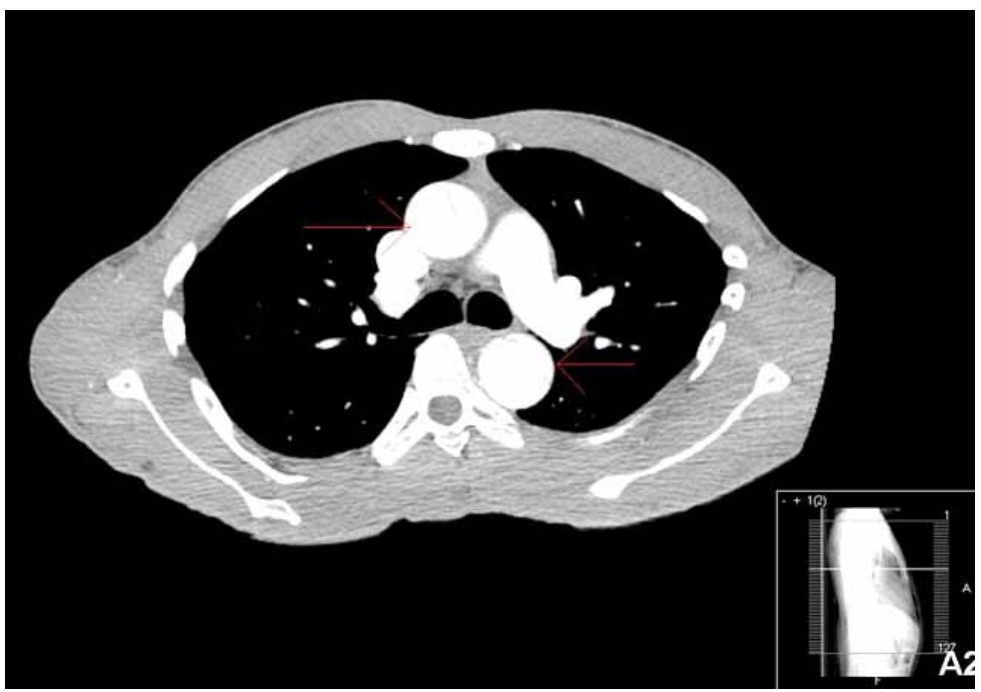

$\mathbf{a}$

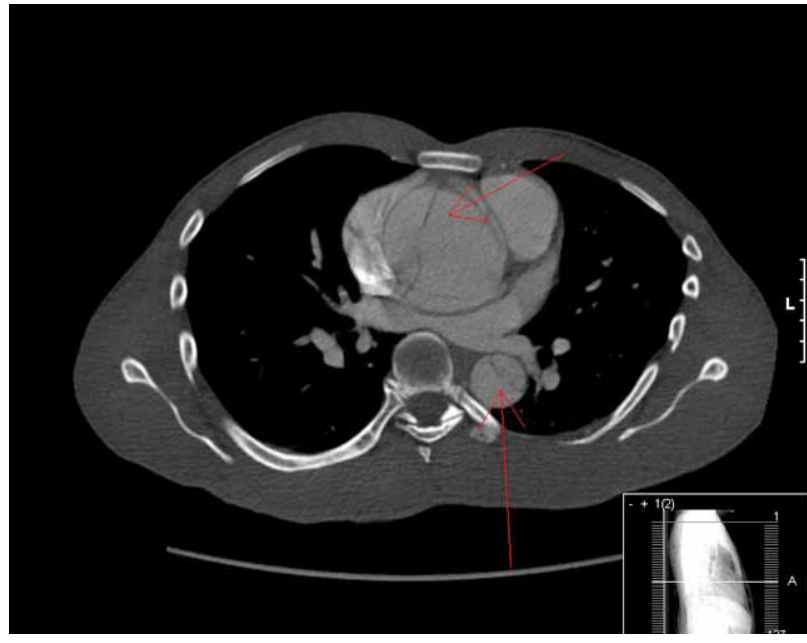

b

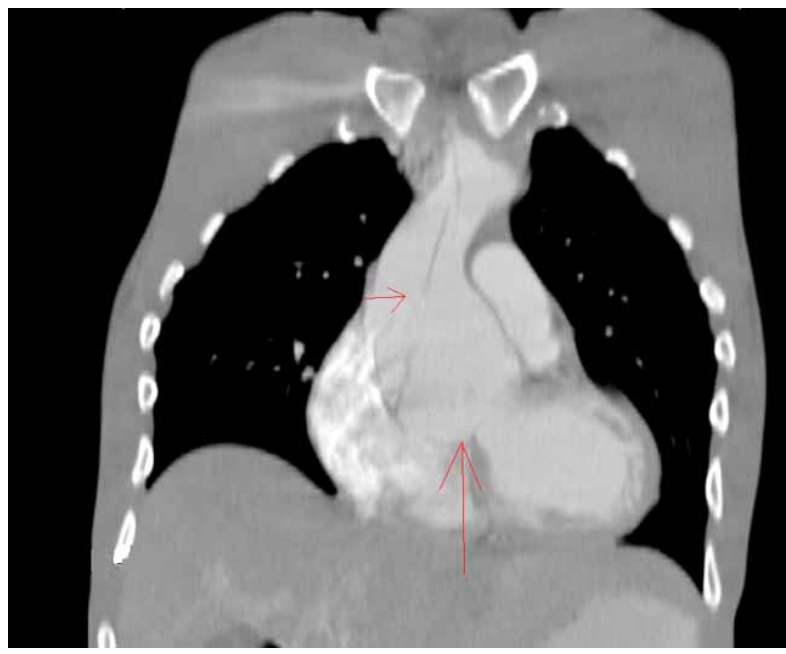

c

Fig. (2). a. CT scan of Chest showing aortic dissection of ascending and descending aorta.

b. CT scan of chest showing both aortic root dilatation with dissection and descending aortic dissection.

c. Coronal view of CT scan of chest showing the dissection of ascending aorta and aortic root dilatation. 
treated surgically, the mortality is $26 \%$, while medically treated Type A dissections have a much higher mortality rate at $58 \%$ [1]. Mortality rate has been reported to rise one percent per hour during the first 48 hours following acute dissection [6].

Treatment of other suspected conditions may delay the diagnosis and may exacerbate propagation of the dissection resulting in ischemic events of end organs and/or limbs. Up to $30 \%$ of patients with aortic dissection will have a delayed diagnosis [7]. The diagnosis of dissection can be made with different imaging techniques, usually computed tomography angiography, transthoracic echocardiography or transesophageal echocardiography [8]. The selection of imaging evaluation depends upon the institutional availability.

Ultrasound has emerged as an excellent non-invasive modality to diagnose aortic emergencies. Bedside ultrasound is being used with increasing frequency by emergency physicians as goal-directed examinations meant to answer specific questions. The sensitivity of bedside transthoracic echocardiography has been reported to be $65-78 \%[9,10]$. While TTE has a low sensitivity, there have been a number of case reports describing the diagnosis of both ascending and descending aortic dissection using bedside ultrasound [11-14]. Emergency bedside ultrasound has been shown to be accurate when performed by physicians with adequate training [15]. An urgent CT angiography scan or TEE can be obtained in a clinically stable patient to confirm and further delineate these findings.

Acute aortic dissection of the ascending aorta is a true surgical emergency. In the presence of a clinical suspicion of any aortic dissection, intravenous beta-blocker therapy should be initiated to control of blood pressure and heart rate with beta-blockers to reduce hemodynamic stress, shearing effect and propagation of dissection [16].

\section{CONCLUSION}

Acute dissection is an uncommon but potentially catastrophic illness with high mortality and its presentation can be varied and subtle. Its prognosis depends critically on the early diagnosis and prompt management and referral. Early clinical suspicion may lead to appropriate imaging studies to confirm the diagnosis and prompt initiation of appropriate medical and/or surgical therapy. This case report underscores the utility of emergency bedside ultrasound that may discover potential aortic catastrophes.

\section{REFERENCES}

[1] Hagan PG, Nienaber CA, Isselbacher EM, et al. The International Registry of Acute Aortic Dissection (IRAD): New insights into an old disease. JAMA 2000; 283: 897.

[2] Spittell PC, Spittell JA, Joyce JW, et al. Clinical features and differential diagnosis of aortic dissection: experience with 236 cases (1980 - 1990). Mayo Clin Proc 1993; 68: 642-51.

[3] Woo KM, Schneider JI. High-risk chief complaints I: chest pain-the big three. Emerg Med Clin North Am 2009; 27(4): 685-712.

[4] DeBakey ME, Henly WS, Cooley DA, Morris GC Jr, Crawford ES, Beall AC Jr. Surgical management of dissecting aneurysms of the aorta. J Thorac Cardiovasc Surg 1965; 49: 130-49.

[5] Daily PO, Trueblood HW, Stinson EB, Wuerflein RD, Shumway NE. Management of acute aortic dissections. Ann Thorac Surg 1970; 10: 237-47.

[6] Hirst AD, Johns VJ, Kime SW. Dissecting aneurysm of the aorta: a review of 505 cases. Medicine 1958; 37: 217-79.

[7] Erbel R, Alfonso F, Boileau C, et al. Diagnosis and management of aortic dissection. Eur Heart J 2001; 22: 1642-81.

[8] Rapezzi C, Longhi S, Graziosi M, et al. Risk factors for diagnostic delay in acute aortic dissection. Am J Cardiol 2008; 102(10): 1399. 406.

[9] Kodolitsch Y, Krause N, Spielmann R, et al. Diagnostic potential of combined trans-thoracic echocardiography and x-ray computed tomography in suspected aortic dissection. Clin Cardiol 1999; 22: 345-52.

[10] Nienaber CA, Von KY, Nicolas V, et al. The diagnosis of thoracic aortic dissection by noninvasive imaging procedures. N Engl J Med 1993; 328(1): $1-9$

[11] Perkins A, Liteplo A, Noble V. Ultrasound diagnosis of type A aortic dissection. J Emerg Med 2010; 38(4): 490-3.

[12] Divakaran V, Mungee S, Salciccioli L, Clark LT. Bedside ultrasound diagnosis of aortic dissection in a cocaine abuser. Eur $\mathbf{J}$ Int Med 2007; 18(8): 595-6

[13] Fojtik JP, Costantino TG, Dean AJ. The diagnosis of aortic dissection by emergency medicine ultrasound. J Emerg Med 2007; 32(2): 191-6.

[14] Kaban J, Raio C. Emergency department diagnosis of aortic dissection by bedside transabdominal ultrasound. Acad Emerg Med 2009, 16(8): 809-10.

[15] Counselman FL, Sanders A, Slovis CM, et al. The status of bedside ultrasonography training in emergency medicine residency programs. Acad Emerg Med 2003; 10(1): 37-42.

[16] Moon MR. Approach to the treatment of aortic dissection. Surg Clin North Am 2009; 89(4): 869-93. 